\title{
CARACTERIZAÇÃO DE HÍBRIDOS DE Poncirus trifoliata E DE OUTROS PORTA-ENXERTOS DE CITROS NO ESTADO DA BAHIA ${ }^{1}$
}

\author{
ORLANDO SAMPAIO PASSOS ${ }^{2}$, LEANDRO SANTOS PEIXOUTO ${ }^{3}$, LEONARDO COSTA DOS SANTOS ${ }^{3}$, \\ RANULFO CORREAA CALDAS ${ }^{2}$, WALTER DOS SANTOS SOARES FILHO ${ }^{2}$
}

\begin{abstract}
RESUMO - Estudos visando à caracterização de híbridos de Poncirus trifoliata (L.) Raf. e porta-enxertos tradicionais foram realizados no sentido de se conhecer o seu comportamento nas condições tropicais do Estado da Bahia. Foram tomadas medidas de altura, diâmetro e peso de frutos, número de sementes por fruto e por quilo, bem como contagem de embriões, taxa de poliembrionia, percentagens de germinação e de vingamento de borbulhas na enxertia, em mais de 34 acessos, a maioria introduzida da Califórnia - EUA. Os dados obtidos estimulam o uso de alguns híbridos de trifoliata, como os das tangerineiras 'Cleópatra' (Citrus reshni hort. ex Tanaka) e 'Sunki' (C. sunki hort. ex Tanaka) com os trifoliatas 'Swingle' e 'English'.
\end{abstract}

Termos para indexação: Características de fruto, poliembrionia, número de embriões por semente, peso de sementes, germinação de semente, vingamento na enxertia.

\section{CHARACTERIZATION OF Poncirus trifoliata HYBRIDS AND OTHER CITRUS ROOTSTOCKS IN THE STATE OF BAHIA}

\begin{abstract}
Studies on characterization of trifoliate hybrids and traditional rootstocks were accomplished in order to know their behavior under tropical conditions in the State of Bahia. Measurements of the height, diameter and weight of the fruits were taken, number of seeds per fruit and per kilo were counted, as well as number of embryos, polyembryony and germination rate and grafting in 34 accessions, mostly introduced from California, USA. The obtained data encourage the use of some trifoliate hybrids, as those from crosses between 'Cleopatra' mandarin (C. reshni hort. ex Tanaka) and 'Sunki' mandarin (C. sunki hort. ex Tanaka) with 'Swingle' and 'English' trifoliate.
\end{abstract}

Index terms: Fruit characteristics, polyembryony, number of embryos per seed, weight of seeds, seed germination rate, budding.

\section{INTRODUÇÃO}

Poncirus trifoliata (L.) Raf., também denominada trifoliata, é um dos porta-enxertos de maior uso na citricultura mundial, ocupando posição de destaque no Japão, Austrália, Nova Zelândia, Argentina, Uruguai e Estados Unidos da América (Califórnia). Essa preferência dá-se em função de certas peculiaridades induzidas por esta espécie às combinações copa/porta-enxerto de que participa, tais como porte anão, tolerância à gomose de Phytophthora, tolerância ao vírus da tristeza e a nematóides, e produção de frutos de excelente qualidade, embora com baixa média de produtividade e baixa tolerância à seca (Castle et al., 1989). Passos et al. (1973) relataram resultados negativos obtidos no Estado da Bahia com o trifoliata, em competição com porta-enxertos tradicionais, tendo como copa laranjeiras-doces [Citrus sinensis (L.) Osbeck]. Híbridos de P. trifoliata com as tangerineiras 'Cleópatra' (C. reshni hort. ex Tanaka) e 'Sunki' $(C$. sunki hort. ex Tanaka), no entanto, mostraram comportamento comparável ao do limoeiro 'Cravo' (C. limonia Osbeck) e de outros porta-enxertos convencionais quando enxertados com laranjeira 'Hamlin' (C. sinensis) (Cunha Sobrinho et al., 1986).

Diversificar o uso de porta-enxertos no Brasil é meta imperiosa, especialmente neste momento em que a citricultura se depara com uma séria ameaça: a morte súbita dos citros (MSC), que afeta pomares enxertados em limoeiro 'Cravo', porta-enxerto mais utilizado no País (Bassanezi et al., 2003).

Desconhecem-se estudos dessa natureza, com variedadesporta-enxerto relacionadas a $P$. trifoliata, no Brasil, à exceção daqueles conduzidos no passado por Vasconcelos \& Araújo (1975), na Baixada Fluminense, visando a determinar o número de sementes por fruto $\mathrm{e}$ peso de sementes de vinte e dois híbridos obtidos pelo Departamento de Agricultura dos Estados Unidos da América - USDA, Indio, Califórnia. O objetivo deste trabalho foi o de avaliar variáveis relacionadas aos frutos, sementes e seedlings (plantas obtidas de sementes) de híbridos de P. trifoliata e de porta-enxertos tradicionais, no sentido de se conhecer o seu comportamento nas condições tropicais do Estado da Bahia.

\section{MATERIAL E MÉTODOS}

O estudo foi realizado no município de Cruz das Almas, Recôncavo Baiano, situado a 12 40' 39" latitude sul e 39 6' $23^{\circ}$ " longitude oeste e altitude de 225 metros. Considerando médias anuais, a temperatura é de $24^{\circ} \mathrm{C}$, a umidade relativa de $80 \%$ e a precipitação pluvial de cerca de $1.200 \mathrm{~mm}$. Os solos são arenosos, classificados como sedimentos terciários da série Barreiras (latossolo vermelho amarelo). As cultivares-porta-enxerto analisadas compreenderam acessos introduzidos da Califórnia - EUA, e de Cordeirópolis, Estado de São Paulo, bem como clones de tangerineira 'Sunki' obtidos pela Embrapa Mandioca e Fruticultura Tropical, a saber: citrangeiros (C. sinensis x $P$. trifoliata) 'Troyer', 'Troyer' 70-150, 'Carrizo', 'Morton', 'Rusk' e 'Yuma', citrumeleiros (C. paradisi Macfad. x $P$. trifolata) 'Swingle', ALP, 'Swingle' 70-133, 'Swingle' CRC 3767, 'Sacaton' 71-79 e 'Tucson' 71-131, P. trifoliata seleção CRC 3551 CN, limoeiro 'Cravo' x P. trifoliata, P. trifoliata x limoeiro 'Cravo', tangerineira 'Cleópatra' x citrangeiro 'Carrizo' 226, 'Cleópatra' x trifoliata 'Barnes' 245, 'Cleópatra' x trifoliata 'Swingle' 287, 'Cleópatra' x trifoliata 'Swingle' 288, 'Cleópatra' x trifoliata 'Swingle' 294, 'Cleópatra' x trifoliata 'Swingle' 305, tangerineira 'Sunki' x trifoliata 'English' 256, 'Sunki' x trifoliata. 'English' 264, 'Sunki’ x trifoliata 'Swingle' 308, 'Sunki' x trifoliata 'Swingle' 314, limoeiros 'Rugoso da Flórida' (C. jambhiri Lush.), 'Cravo Maratá' e 'Volkameriano' $(C$. volkameriana V. Ten. \& Pasq.), tangerineiras 'Cleópatra', 'Sunki', 'Sunki Maravilha' e 'Sunki Tropical', além dos híbridos limoeiro 'Cravo' x tangerineira 'Cleópatra' e 'Cleópatra' x 'Cravo' .

Quinze frutos de cada acesso foram colhidos no Banco Ativo de Germoplasma de Citros da Embrapa Mandioca e Fruticultura Tropical, em junho de 2003, tendo sido adotada a seguinte seqüência: 1. Tomada de altura, diâmetro (por meio de paquímetro) e peso de 10 frutos; 2. Contagem do número de sementes por fruto, peso das sementes e número de sementes por quilo; 3 . Os cinco frutos colhidos

\footnotetext{
${ }^{1}$ (Trabalho 32-2006). Recebido: 21-03-2006. Aceito para publicação: 25-08-2006.

2 Pesquisadores da Embrapa Mandioca e Fruticultura Tropical, C.P. 007, 44380-000 Cruz das Almas - BA

${ }^{3}$ Alunos do Centro de Ciências Agrárias e Ambientais da Universidade Federal da Bahia, 44380-000 Cruz das Almas - BA
} 
restantes destinados à contagem de embriões foram transportados para o Laboratório de Cultura de Tecidos e lavados em água corrente. Em cada fruto, efetuou-se um corte transversal incompleto, para não danificar as sementes, que foram extraídas e lavadas com solução de água e detergente, secadas e despojadas manualmente do tegumento externo (testa). Sob microrcópio estereoscópico (40X), com auxílio de pinça e bisturi, as sementes foram cortadas longitudinalmente, respeitando-se certa distância da região da micrópila para não provocar lesões nos embriões. Procedeu-se então à eliminação do tegumento interno (tégmen), sendo os embriões extraídos, incluindo os cotilédones, e agrupados nas seguintes quatro classes de tamanho: a) embriões muito pequenos (MP), com menos de $1,0 \mathrm{~mm}$ de comprimento; b) embriões pequenos (P), com 1,0 mm a 2,9 $\mathrm{mm}$ de comprimento; c) embriões médios (M), com 3,0 mm a 4,9 mm de comprimento, e d) embriões grandes $(\mathrm{G})$, maiores ou iguais a $5,0 \mathrm{~mm}$ de comprimento, seguida da determinação da taxa de poliembrionia, esta obtida pela expressão: número de sementes com mais de um embrião x 100 / número total de sementes; 4. Em ambiente protegido com tela antiafídica, as sementes (10 frutos) foram postas para germinar em tubetes ( $3 \mathrm{~cm}$ de diâmetro x $12 \mathrm{~cm}$ de comprimento), com substrato de casca de pínus, em número de 86 sementes para cada cultivar, analisando-se as percentagens de germinação aos 45 dias após a semeadura; 5. A altura e diâmetro dos "cavalinhos" foram medidos aos três meses (época do tranplante) e 10 meses (época da enxertia) após a semeadura; 6 . A percentagem de vingamento das enxertias (número de brotações de borbulhas enxertadas x 100 / total de borbulhas enxertadas) foi quantificada aos 40 dias após a enxertia, considerando-se quatro diferentes cultivares-copa \{tangerineirastangeleiro [tangerineira 'Clementina' ( $C$. clementina hort. ex Tanaka) x tangelo 'Orlando' (pomeleiro 'Duncan' $C$. paradisi x tangerineira 'Dancy' C. tangerina hort. ex Tanaka)] Nova e Robinson, laranjeira Pêra e limeira ácida Tahiti [C. latifolia (Yu. Tanaka) Tanaka]\} e observando-se o delineamento experimental inteiramente casualizado; os distintos porta-enxertos foram enxertados com 10 borbulhas de cada variedade-copa, pelo método da borbulhia; o "cavalo" foi curvado e, 15 dias após a enxertia, foi cortado a $1 \mathrm{~cm}$ acima do ponto da enxertia. Aos dados foram aplicados procedimentos da estatística descritiva e calculado a correlação linear simples entre as variáveis número de sementes/fruto e número de sementes/quilo.

\section{RESULTADOS E DISCUSSÃO}

Levando-se em conta o número de sementes por fruto, um dos caracteres mais importantes na avaliação de um porta-enxerto, os seguintes acessos (com número de sementes por fruto superior à media geral dos porta-enxertos analisados) merecem destaque: citrangeiros 'Troyer' 70-150 e 'Yuma', citrumeleiros 'Swingle', 'Swingle 70-133', 'Tucson' 71-131 e 'Swingle' CRC 3767', híbridos $P$. trifoliata x limoeiro 'Cravo', limoeiro 'Cravo' x P. trifoliata, 'Cleópatra' $\mathrm{x}$ limoeiro ‘Cravo', 'Cleópatra' x trifoliata 'Barnes’ 245, tangerineira

TABELA 1 - Altura, diâmetro e peso do fruto, número de sementes por fruto e por quilo de sementes, relativos a porta-enxertos de citros nas condições de Cruz das Almas - BA.

\begin{tabular}{|c|c|c|c|c|c|c|}
\hline Variedades -porta-enxerto & $\begin{array}{c}\text { Altura } \\
(\mathrm{cm})\end{array}$ & $\begin{array}{l}\text { Diâmetro } \\
(\mathrm{cm})\end{array}$ & $\begin{array}{c}\text { Peso } \\
(\mathrm{g})\end{array}$ & $\begin{array}{l}\text { Sementes/ } \\
\text { fruto }\end{array}$ & $\begin{array}{c}\text { Peso } 100 \\
\text { sem. }(\mathrm{g})\end{array}$ & $\begin{array}{c}\text { Sementes } \\
\text { em } 1 \mathrm{~kg}\end{array}$ \\
\hline \multicolumn{7}{|c|}{ Poncirus trifoliata e híbridos } \\
\hline Citrange Carrizo & $4,6 a$ & 4,7 & 59,6 & 12,8 & 10,50 & 9.524 \\
\hline Citrange Morton & 4,0 & 4,7 & 43,6 & 15,3 & 16,60 & 6.024 \\
\hline Citrange Rusk & $4,9 \mathrm{a} \mathrm{b}$ & $5,2 \mathrm{a} \mathrm{b}$ & 64,2 & 9,0 & 25,61 & 3.905 \\
\hline Citrange Troyer & $4,4 a$ & 4,7 & 53,0 & 13,2 & 5,87 & 17.036 \\
\hline Citrange Troyer $70-150$ & $4,8 \mathrm{a} b$ & $5,0 \mathrm{a} \mathrm{b}$ & 62,6 & $20,4 \mathrm{a} \mathrm{b}$ & 15,00 & 6.667 \\
\hline Citrange Yuma & $5,3 a b$ & $5,7 \mathrm{a} \mathrm{b}$ & 94,6 & $19,2 \mathrm{a} \mathrm{b}$ & 17,34 & 5.767 \\
\hline Citrumelo ALP & $5,0 \mathrm{a} \mathrm{b}$ & $5,8 \mathrm{a} \mathrm{b}$ & 73,0 & 7,4 & 17,59 & 5.685 \\
\hline Citrumelo Sacaton 71-79 & $5,4 a b$ & $5,0 \mathrm{a} \mathrm{b}$ & 51,0 & 13,7 & 0 & 0 \\
\hline Citrumelo Swingle & $5,2 \mathrm{a} \mathrm{b}$ & $5,3 \mathrm{a} \mathrm{b}$ & 71,1 & $24,7 \mathrm{a} \mathrm{b}$ & 22,80 & 4.386 \\
\hline Citrumelo Swingle 70-133 & $6,7 \mathrm{a} b$ & $6,2 \mathrm{a} \mathrm{b}$ & 124,6 & $29,9 a \mathrm{~b}$ & 18,06 & 5.537 \\
\hline Citrumelo Swingle CRC3767 & $6,5 \mathrm{a} \mathrm{b}$ & $6,5 \mathrm{a} \mathrm{b}$ & 124,5 & $20,9 a \mathrm{~b}$ & 18,46 & 5.417 \\
\hline Citrumelo Tucson 71-131 & 2,8 & 3,2 & 24,2 & 8,4 & 4,90 & 20.408 \\
\hline P. trifoliata $\mathrm{x}$ limoeiro Cravo & 3,4 & 3,9 & 27,3 & $17,3 \mathrm{a} \mathrm{b}$ & 16,02 & 6.242 \\
\hline P. trifoliata CRC3551 CN & 3,6 & 4,1 & 31,2 & 8,7 & 22,45 & 4.454 \\
\hline \multicolumn{7}{|c|}{ Limoeiros e híbridos } \\
\hline Rugoso da Flórida & $6,8 \mathrm{a} \mathrm{b}$ & $7,2 \mathrm{a}$ & 172,8 & 9,5 & 9,03 & 11.074 \\
\hline Volkameriano & $5,8 \mathrm{a} \mathrm{b}$ & $6,0 \mathrm{a}$ & 113,3 & $13,0 \mathrm{~b}$ & 11,25 & 8.889 \\
\hline Cravo Maratá & $5,9 a \mathrm{~b}$ & $6,0 \mathrm{a}$ & 104,2 & $13,8 \mathrm{~b}$ & 28,19 & 3.547 \\
\hline Cravo x $P$. trifoliata & 3,0 & 3,5 & 18,6 & $15,8 \mathrm{a} \mathrm{b}$ & 10,84 & 9.225 \\
\hline Cravo $\mathrm{x}$ tangerineira Cleópatra & $4,7 \mathrm{a}$ & $5,2 \mathrm{a}$ & 70,9 & 9,4 & 5,09 & 19.646 \\
\hline \multicolumn{7}{|c|}{ Tangerineiras e híbridos } \\
\hline Cleópatra & $4,0 \mathrm{~b}$ & $5,5 \mathrm{a} \mathrm{b}$ & 63,2 & 10,3 & 10,71 & 9.337 \\
\hline Sunki & 3,1 & 4,0 & 25,7 & 9,0 & 5,2 & 19.231 \\
\hline Sunki Maravilha & 2,3 & 2,8 & 10,6 & 10,8 & 5,26 & 19.011 \\
\hline Sunki Tropical & 3,2 & 4,0 & 27,7 & $19,5 \mathrm{a} \mathrm{b}$ & 6,55 & 15.267 \\
\hline Cleópatra x citr.Carrizo226 & $5,1 \mathrm{a} \mathrm{b}$ & $6,1 \mathrm{a} \mathrm{b}$ & 93,1 & 13,0 & 17,59 & 5.685 \\
\hline Cleópatra $\mathrm{x}$ limoeiro Cravo & $3,8 \mathrm{~b}$ & $4,4 \mathrm{~b}$ & 39,4 & $17,1 \mathrm{a} \mathrm{b}$ & 7,12 & 13.966 \\
\hline Cleópatra $\mathrm{x}$ trif. Barnes 245 & $3,8 \mathrm{~b}$ & 4,0 & 34,9 & $27,4 a b$ & 13,31 & 7.513 \\
\hline Cleópatra $\mathrm{x}$ trif Swingle 287 & $3,6 \mathrm{~b}$ & 4,1 & 32,3 & $19,6 \mathrm{a} \mathrm{b}$ & 13,09 & 7.639 \\
\hline Cleópatra $\mathrm{x}$ trif. Swingle 288 & 2,8 & 3,0 & 13,4 & 5,5 & 10,85 & 9.217 \\
\hline Sunki x trif Swingle 256 & 3,5 & 4,1 & 31,9 & $22,6 \mathrm{a} \mathrm{b}$ & 19,84 & 5.040 \\
\hline Sunki x trif. Swingle 264 & $4,0 \mathrm{~b}$ & $4,6 \mathrm{~b}$ & 47,2 & $26,5 \mathrm{a} b$ & 18,22 & 5.488 \\
\hline Sunki x trif. Swingle 308 & 3,2 & 3,7 & 24,9 & 14,4 & 5,35 & 18.692 \\
\hline Sunki x trif. Swingle 314 & $3,7 \mathrm{~b}$ & $4,2 \mathrm{~b}$ & 36,3 & $17,6 \mathrm{a} \mathrm{b}$ & 15,04 & 6.649 \\
\hline
\end{tabular}

$\mathrm{a}=$ cultivares que se encontram acima da média geral

$\mathrm{b}=$ cultivares que se encontram acima da média do grupo 
TABELA 2 - Número de embriões, de sementes e taxa de poliembrionia de porta-enxertos de citros nas condições de Cruz das Almas-BA

\begin{tabular}{|c|c|c|c|c|}
\hline \multirow{3}{*}{ Variedades -porta-enxerto } & \multirow{2}{*}{$\begin{array}{l}\mathrm{N}^{\mathrm{o}} \mathrm{de} \\
\text { frutos }\end{array}$} & \multicolumn{2}{|c|}{ Embriões } & \multirow{2}{*}{$\begin{array}{c}\text { Taxa de } \\
\text { poliembrionia (\%) }\end{array}$} \\
\hline & & Total & Embr./sem. & \\
\hline & \multicolumn{3}{|c|}{ Híbridos de Poncirus trifoliata } & \\
\hline Citrange Morton & 3 & 311 & $5,87 \mathrm{ab}$ & 86,79 \\
\hline Citrange Rusk & 5 & 525 & $5,05 \mathrm{ab}$ & 92,30 \\
\hline Citrange Troyer $70-150$ & 5 & 709 & $6,95 \mathrm{a} b$ & 95,09 \\
\hline Citrange Yuma & 5 & 110 & 1,36 & 21,00 \\
\hline Citrumelo ALP & 5 & 102 & 1,67 & 34,42 \\
\hline Citrumelo Swingle & 5 & 364 & 2,96 & 65,04 \\
\hline Citrumelo Swingle 70-133 & 5 & 612 & 3,66 & 83,23 \\
\hline Citrumelo Swingle CRC3767 & 4 & 113 & 1,69 & 38,80 \\
\hline Citrumelo Tucson 71-131 & 5 & 64 & 1,36 & 12,76 \\
\hline P. trifoliata $\mathrm{x}$ limoeiro Cravo & 4 & 715 & $8,41 \mathrm{ab}$ & 98,82 \\
\hline Cravo x P. trifoliata & 5 & 535 & $7,43 \mathrm{ab}$ & 97,22 \\
\hline \multicolumn{5}{|c|}{ Limoeiros e híbridos } \\
\hline Rugoso da Flórida & 5 & 386 & $4,89 \mathrm{~b}$ & 96,20 \\
\hline Volkameriano & 5 & 357 & 2,57 & 53,23 \\
\hline Cravo Maratá & 4 & 125 & 1,87 & 43,28 \\
\hline Cravo $\mathrm{x}$ tangerineira Cleópatra & 5 & 163 & 1,87 & 50,57 \\
\hline \multicolumn{5}{|c|}{ Tangerineiras e híbridos } \\
\hline Sunki & 5 & 74 & 1,80 & 66,21 \\
\hline Sunki Maravilha & 1 & 40 & $10,00 \mathrm{a} \mathrm{b}$ & 100 \\
\hline Sunki Tropical & 5 & 638 & $7,88 \mathrm{a} b$ & 99,98 \\
\hline Cleópatra & 5 & 825 & $8,01 \mathrm{ab}$ & 97,08 \\
\hline Cleópatra x citr. Carrizo226 & 6 & 575 & $5,81 \mathrm{a}$ & 90,00 \\
\hline Cleópatra $\mathrm{x}$ limoeiro Cravo & 5 & 738 & $9,97 a b$ & 100 \\
\hline Cleópatra $\mathrm{x}$ trif. Barnes 245 & 4 & 779 & $11,29 \mathrm{a} b$ & 100 \\
\hline Cleópatra $\mathrm{x}$ trif. Swingle 287 & 5 & 582 & $8,82 \mathrm{a} \mathrm{b}$ & 90,90 \\
\hline Cleópatra x trif. Swingle 294 & 5 & 366 & 4,58 & 85,00 \\
\hline Cleópatra $\mathrm{x}$ trif. Swingle 305 & 2 & 537 & $7,26 \mathrm{a}$ & 94,59 \\
\hline Sunki $x$ trif. Swingle 256 & 5 & 1112 & $9,42 a b$ & 99,15 \\
\hline Sunki $x$ trif. Swingle 264 & 5 & 603 & $8,15 \mathrm{ab}$ & 97,29 \\
\hline Sunki $x$ trif. Swingle 308 & 5 & 559 & $6,90 \mathrm{a}$ & 91,35 \\
\hline Sunki $\mathrm{x}$ trif. Swingle 314 & 5 & 460 & $5,97 \mathrm{a}$ & 96,10 \\
\hline
\end{tabular}

$\mathrm{a}=$ cultivares que se encontram acima da média geral

$\mathrm{b}=$ cultivares que se encontram acima da média do grupo

'Sunki' x trifoliata 'English' 256, 'Sunki' x trifoliata 'Swingle' 314, 'Sunki' x trifoliata 'English' 264, 'Cleópatra' x trifoliata 'Swingle' 287, além da tangerineira 'Sunki Tropical'. Observou-se uma tendência de associação negativa e significativa(-0,3868) entre o número de sementes por fruto e o número de sementes por quilo, o que indica uma tendência de associação positiva entre o número de sementes por fruto e o tamanho da semente, como mostram as cultivares citrangeiros Morton, Rusk, Troyer 70-150 e Yuma, citrumeleiros Swingle, Swingle 70-133 e Swingle CRC3767, P. trifoliata x limoeiro Cravo, tangerineira Cleópatra x citrangeiro Carrizo 226, tangerineira Sunki x trifoliata English 256, Sunki x trifoliata Swingle 314, limoeiro Cravo Maratá e tangerineira Sunki Tropical (Tabela 1).

A taxa de poliembrionia é outro caráter de extrema importância na escolha de um porta-enxerto comercial, a qual, quanto mais elevada, aumentam as chances de um porta-enxerto, quando propagado por semente, dar formação a "cavalinhos" de origem nucelar, semelhantes, portanto, à cultivar-mãe. No presente caso, observaram-se taxas superiores a $90 \%$ nos híbridos citrangeiros 'Rusk' e ‘Troyer', P. trifoliata x limoeiro ‘Cravo', 'Cravo’ x P. trifoliata, tangerineira ‘Cleópatra' x trifoliata 'Barnes' 245, ‘Cleópatra’ x citrange 'Carrizo' 226, 'Cleópatra' x trifoliata 'Swingle' 287, 'Cleópatra' x trifoliata 'Swingle' 305, 'Cleópatra' x limoeiro 'Cravo', tangerineira 'Sunki' x trifoliata 'English' 256, 'Sunki' x trifoliata 'English' 264, 'Sunki' x trifoliata 'Swingle' 308, 'Sunki' x trifoliata 'Swingle' 314, além do limoeiro 'Rugoso da Flórida' e das tangerineiras 'Sunki Maravilha', 'Sunki Tropical' e 'Cleópatra' (Tabela 2). Quanto ao número de embriões por semente, verificou-se superioridade (> 5) em diversos híbridos de trifoliata e nas tangerineiras 'Cleópatra', 'Sunki Maravilha' e 'Sunki Tropical' (Tabela 2).

No que diz respeito à taxa de germinação, apesar das variações constatadas, ficou evidente que o período de germinação está entre 45 e 60 dias após a semeadura.

Como era esperado, os porta-enxertos tradicionais (limoeiros 'Cravo Maratá', 'Rugoso da Flórida' e 'Volkameriano') apresentaram maior vigor após 3 e 10 meses de transplantados. A percentagem de vingamento de enxertos também foi relativamente alta entre os portaenxertos tradicionais, incluindo a tangerineira 'Cleópatra', o mesmo verificando-se em relação aos híbridos citrumeleiro 'Swingle', limoeiro 
TABELA 3 - Percentagem de germinação, altura e diâmetro do caule dos "cavalinhos" aos 3 e 10 meses de transplante e percentagem de vingamento de enxertos 40 dias após a enxertia com a laranjeira 'Pêra', tangeleiros 'Nova' e 'Robinson' e limeira ácida 'Tahiti', sob telado, nas condições de Cruz das Almas - BA.

\begin{tabular}{|c|c|c|c|c|c|c|c|c|}
\hline \multirow{2}{*}{ Variedades -porta-enxerto } & \multirow{2}{*}{$\begin{array}{c}\text { \% de } \\
\text { germinação } \\
45 \text { dias após a } \\
\text { semeadura }\end{array}$} & \multirow{2}{*}{$\begin{array}{l}\text { Altura }(\mathrm{cm}) \\
\text { três meses } \\
\text { após o } \\
\text { transplante }\end{array}$} & Altura & Diâmetro & \multicolumn{4}{|c|}{$\begin{array}{c}\text { \% de vingamento do enxerto } \\
40 \text { dias após a enxertia }\end{array}$} \\
\hline & & & \multicolumn{2}{|c|}{$\begin{array}{c}10 \text { meses após o } \\
\text { transplante }\end{array}$} & Nova & Robinson & Pêra & Tahiti \\
\hline \multicolumn{9}{|l|}{ Poncirus trifoliata e híbridos } \\
\hline Citrange Carrizo & 74 & 7,1 & 89 & 0,07 & 44 & 0 & 44 & 25 \\
\hline Citrange Morton & 65 & 6,9 & 93 & 0,04 & 0 & 38 & 25 & 27 \\
\hline Citrange Rusk & 70 & 7,2 & 74 & 0,06 & 33 & 33 & 50 & 43 \\
\hline Citrange Troyer & 45 & 8,1 & 84 & 0,07 & 40 & 40 & 40 & 17 \\
\hline Citrange Troyer 70-150 & 92 & 7,8 & 82 & 0,07 & 50 & 50 & 50 & 60 \\
\hline Citrange Yuma & 76 & 7,1 & 37 & 0,06 & 0 & 25 & 0 & 0 \\
\hline Citrumelo ALP & 35 & 8,8 & 121 & 0,07 & 67 & 78 & 89 & 100 \\
\hline Citrumelo Swingle & 64 & 6,6 & 62 & 0,06 & 100 & 20 & 60 & 40 \\
\hline Citrumelo Swingle 70-133 & 77 & 8,4 & 64 & 0,06 & 100 & 50 & 67 & 33 \\
\hline Citrumelo Swingle CRC3767 & 86 & 7,4 & 48 & 0,07 & 80 & 60 & 67 & 33 \\
\hline Citrumelo Tucson 71-131 & 76 & 4,9 & 37 & 0,04 & 0 & 0 & 0 & 0 \\
\hline P. trifoliata $\mathrm{x}$ limoeiro Cravo & 44 & 5,6 & 63 & 0,06 & 0 & 25 & 0 & 0 \\
\hline Cravo $\times P$. trifoliata & 47 & 6,5 & 43 & 0,06 & 0 & 0 & 0 & 0 \\
\hline P. trifoliata CRC3551 CN & 81 & 6,3 & 67 & 0,06 & 0 & 14 & 29 & 29 \\
\hline \multicolumn{9}{|l|}{ Limoeiros e híbridos } \\
\hline Rugoso da Flórida & 74 & 7,3 & 138 & 0,09 & 89 & 78 & 90 & 89 \\
\hline Cravo Maratá & 84 & 6,4 & 140 & 0,1 & 100 & 50 & 70 & 67 \\
\hline Cravo $\mathrm{x}$ tangerineira Cleópatra & 57 & 6,3 & 101 & 0,08 & 80 & 100 & 80 & 80 \\
\hline Volkameriano & 88 & 7,8 & 100 & 0,09 & 100 & 67 & 88 & 100 \\
\hline \multicolumn{9}{|l|}{ Tangerineiras e híbridos } \\
\hline Cleópatra & 67 & 6,6 & 72 & 0,07 & 75 & 75 & 25 & 100 \\
\hline Sunki & 93 & 6,4 & 70 & 0,06 & 43 & 29 & 25 & 25 \\
\hline Sunki Maravilha & 87 & 5,5 & 75 & 0,07 & 63 & 71 & 63 & 29 \\
\hline Sunki Tropical & 40 & 6,3 & 71 & 0,07 & 0 & 56 & 0 & 0 \\
\hline Cleópatra x citr. Carrizo 226 & 80 & 7,9 & 85 & 0,06 & 60 & 0 & 20 & 100 \\
\hline Cleópatra x limoeiro Cravo & 78 & 3,7 & 66 & 0,06 & 0 & 50 & 0 & 0 \\
\hline Cleópatra x trif. Barnes 245 & 55 & 7,7 & 88 & 0,06 & 50 & 50 & 25 & 29 \\
\hline Cleópatra x trif. Swingle 294 & 95 & 7,5 & 84 & 0,06 & 75 & 63 & 75 & 63 \\
\hline Cleópatra x trif. Swingle 287 & 38 & 7,2 & 79 & 0,05 & 60 & 60 & 67 & 60 \\
\hline Cleópatra x trif. Swingle 288 & 35 & 4,5 & 85 & 0,07 & 60 & 67 & 60 & 40 \\
\hline Cleópatra x trif. Swingle 305 & 66 & 6,7 & 84 & 0,06 & 57 & 71 & 83 & 63 \\
\hline Sunki x trif. Swingle 256 & 80 & 7,4 & 98 & 0,06 & 56 & 44 & 67 & 56 \\
\hline Sunki x trif. Swingle 264 & 73 & 9 & 63 & 0,07 & 40 & 20 & 13 & 29 \\
\hline Sunki $x$ trif. Swingle 308 & 82 & 4,9 & 92 & 0,07 & 43 & 29 & 33 & 67 \\
\hline Sunki $x$ trif. Swingle 314 & 83 & 7,1 & 72 & 0,06 & 63 & 29 & 63 & 43 \\
\hline
\end{tabular}

'Cravo' x tangerineira 'Cleópatra', 'Cleópatra' x trifoliata 'Swingle' 287, 'Cleópatra' x trifoliata 'Swingle' 294 e 'Cleópatra' x trifoliata 'Swingle' 305 (Tabela 3).

\section{CONCLUSÕES}

Os estudos evidenciaram a superioridade dos híbridos de trifoliata tangerineira 'Sunki' x trifoliata 'English' 256, tangerineira 'Sunki' x trifoliata 'English' 264 e tangerineira 'Sunki' x trifoliata 'Swingle' 314, entre outros, em relação aos porta-enxertos tradicionais limoeiro ‘Cravo' e limoeiro 'Volkameriano', principalmente no que diz respeito ao número de sementes por fruto, número de embriões por semente e taxa de poliembrionia. A importância desses dados está no fato de que o uso do limoeiro 'Cravo' nos pomares brasileiros é quase exclusivo, o que torna a citricultura vulnerável devido à ameaça de algumas doenças, evidenciando a necessidade de que sejam oferecidas outras cultivares, destacando-se aquelas derivadas de Poncirus trifoliata, porta-enxerto de reconhecidas qualidades horticulturais, como redução de porte, resistência a doenças e indução de frutos de alta qualidade às copas de diversas variedades cítricas. Por se tratar de plantas com melhor adaptação às condições subtropicais ou temperadas, híbridos de trifoliata são mais indicados para condições tropicais.

\section{REFERÊNCIAS}

BASSANEZI, R.B.; FERNANDES, N.G.; YAMAMOTO, P.T. Morte súbita dos citros. Araraquara: Fundecitrus, 2003. p.54.

CASTLE, W.S.; TUCKER, D.P.H.; KREZDORN, A.H.; YOUTSEY, C.O. Rootstock selection: the first step to success. Rootstocks for Florida citrus. Gainsville: University of Florida, Gainesville,1989. p.47.

CUNHA SOBRINHO, A.P. da; PASSOS, O.S.; SOARES FILHO, W. dos S. Diversificação de porta-enxertos na citricultura do Nordeste. In: SEMINÁRIO DE TECNOLOGIAAGROPECUÁRIA INOVADORA PARA O NORDESTE, 1986, Fortaleza. Anais... p.228-234.

PASSOS, O.S.; CUNHA SOBRINHO, A.P.; COELHO, Y.S. O Poncirus trifoliata (L.) Raf. no Estado da Bahia. In: CONGRESSO BRASILEIRO DE FRUTICULTURA, 2., 1973, Viçosa. Anais... p.149-157.

VASCONCELOS, H.O.; ARAÚJO, C.M. Informações preliminares sobre o comportamento de 22 híbridos americanos de portaenxertos para citros na Baixada Fluminense. In: CONGRESSO BRASILEIRO DE FRUTICULTURA, 3., 1975, Rio de Janeiro. Anais... p. 339-348. 